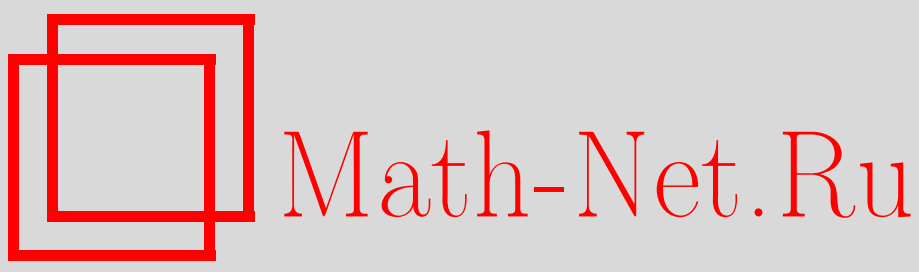

А. Ю. Колесов, Н. Х. Розов, Многочастотный параметрический резонанс в нелинейном волновом уравнении, Изв. РАН. Сер. матем., 2002, том 66, выпуск 6, 49-64

DOI: https://doi.org/10.4213/im409

Использование Общероссийского математического портала Math-Net.Ru подразумевает, что вы прочитали и согласны с пользовательским соглашением http://www. mathnet.ru/rus/agreement

Параметры загрузки:

IP: 54.80 .73 .141

26 апреля 2023 г., $18: 11: 27$ 
УДК 517.926

\author{
А. Ю. Колесов, Н. Х. Розов
}

\title{
Многочастотный параметрический резонанс в нелинейном волновом уравнении
}

Рассматривается краевая задача

$$
u_{t t}+\varepsilon u_{t}+\left(1+\varepsilon \sum_{k=1}^{m} \alpha_{k} \cos 2 \varphi_{k}\right) u=a^{2} u_{x x}-u^{2} u_{t},\left.\quad u\right|_{x=0}=\left.u\right|_{x=\pi}=0
$$

где $0<\varepsilon \ll 1, a>0, \varphi_{k}=\sigma_{k} t+c_{k}, k=1, \ldots, m$. Показывается, что при подходящем выборе натурального $m$ и вещественных параметров $\alpha_{k}, \sigma_{k}$, $k=1, \ldots, m$, можно добиться существования у нее любого фиксированного числа экспоненциально устойчивых квазипериодических по $t$ решений, бифурцирующих из нуля при $\varepsilon>0$.

Библиография: 10 наименований.

Физические основы теории параметрических колебаний представлены в классических работах Л. И. Мандельштама и Н. Д. Папалекси, а математический аспект проблемы изложен, например, в монографиях [1]-[3], содержащих теорию параметрического резонанса как для обыкновенных дифференциальных уравнений, так и для некоторых классов волновых уравнений. Следует отметить еще работы [4], [5], в которых в линейной постановке изучался параметрический резонанс в случае квазипериодической внешней силы.

В настоящей статье рассматривается новый в данной тематике вопрос о нелинейных эффектах, вызванных многочастотным параметрическим внешним воздействием. Основная идея здесь заключается в следуюшем. Пусть имеется некоторая потенциально автоколебательная нелинейная система с достаточно большим набором собственных частот $\omega_{k}, k=1, \ldots, m$, причем эта система недовозбуждена: ее нулевое состояние равновесия экспоненциально устойчиво. Предположим, далее, что на такую систему начинает действовать многочастотная параметрическая внешняя сила с частотами, близкими к $2 \omega_{k_{s}}, s=1, \ldots, n$, где $1 \leqslant k_{1}<k_{2}<\cdots<k_{n} \leqslant m-$ произвольно фиксированный набор индексов. В результате нулевое решение этой системы может стать неустойчивым сразу на всех рассматриваемых собственных частотах $\omega_{k_{s}}, s=1, \ldots, n$, т.е. может наступить так называемый многочастотный параметрический резонанс.

Описанную идею нетрудно реализовать в случае многомерных систем обыкновенных дифференциальных уравнений. Однако наиболее естественным представляется исследование явления многочастотного параметрического резонанса в нелинейных волновых уравнениях, обладающих бесконечным набором собственных

Работа выполнена при поддержке РФФИ (грант № 02-01-01286). 
частот. В связи с этим ниже рассматривается простейшая нелинейная гиперболическая краевая задача и показывается, что посредством многочастотного параметрического внешнего воздействия можно возбудить в ней любое наперед заданное число устойчивых одномодовых квазипериодических по времени колебательных режимов. Более того, за счет подходящего выбора параметров внешнего воздействия любыми можно сделать и номера возбуждаемых мод.

1. Постановка задачи и описание результата. На отрезке $0 \leqslant x \leqslant \pi$ рассмотрим краевую задачу

$$
u_{t t}+\varepsilon u_{t}+[1+\varepsilon f(\varphi)] u=a^{2} u_{x x}-u^{2} u_{t},\left.\quad u\right|_{x=0}=\left.u\right|_{x=\pi}=0
$$

Здесь $\varepsilon>0$ - мальй параметр, $a=\mathrm{const}>0, \varphi=\left(\varphi_{1}, \ldots, \varphi_{m}\right)$, внешняя параметрическая сила имеет вид

$$
f(\varphi)=\sum_{k=1}^{m} \alpha_{k} \cos 2 \varphi_{k}, \quad \varphi_{k}=\sigma_{k} t+c_{k}, \quad k=1, \ldots, m
$$

а натуральное $m$ и наборы вешественных постоянных $\alpha_{k}, \sigma_{k}, c_{k}, k=1, \ldots, m$, произвольно фиксированы. Зададимся, далее, произвольным набором натуральных чисел $n_{1}<n_{2}<\cdots<n_{m}$ и потребуем, чтобы в краевой задаче (1) параметрический резонанс наблюдался на модах $\sin n x$ с номерами $n=n_{k}, k=1, \ldots, m$. Добиться этого можно за счет подходящего выбора фигурирующих в (2) параметров $\alpha_{k}, \sigma_{k}, k=1, \ldots, m$. Способ такого выбора описывается ниже.

Отбросим в (1) нелинейность, а затем применим к получившейся линейной краевой задаче метод Фурье по системе функций $\sin n x, n=1,2, \ldots$ В результате убедимся, что устойчивость нулевого решения задачи (1) определяют характеристические показатели линейных уравнений

$$
\ddot{u}+\varepsilon \dot{u}+\left[\omega_{n}^{2}+\varepsilon f(\varphi)\right] u=0, \quad n=1,2, \ldots,
$$

где $\omega_{n}=\sqrt{1+a^{2} n^{2}}$, а дифференцирование проводится по $t$. Будем считать, далее, что частоты $\sigma_{k}, k=1, \ldots, m$, параметрического внешнего воздействия $(2)$ задаются равенствами

$$
\sigma_{k}=\left(1+\varepsilon \delta_{k}\right) \omega_{n_{k}}, \quad k=1, \ldots, m
$$

где $\delta_{k}, k=1, \ldots, m,-$ некоторые фиксированные постоянные, связанные с амплитудами $\alpha_{k}, k=1, \ldots, m$, соотношениями

$$
\alpha_{k}^{2} /\left(4 \omega_{n_{k}}^{2}\right)-4 \delta_{k}^{2} \omega_{n_{k}}^{2}-1>0, \quad k=1, \ldots, m
$$

Тогда, как следует, например, из содержашихся в [1], [2] результатов, каждое уравнение (3) при $n=n_{k}, k=1, \ldots, m$, имеет экспоненщиально растушее решение. При $n \neq n_{k}$ все решения уравнений (3) при $t \rightarrow \infty$ экспоненциально затухают.

Итак, при условиях (4), (5) в рамках краевой задачи (1) реализуется многочастотный параметрический резонанс: нулевое решение этой задачи неустойчиво на 
модах $\sin n_{k} x, k=1, \ldots, m$. Поэтому естественным образом возникают вопросы о сушествовании и устойчивости квазипериодических по $t$ решений задачи (1), бифурцируюших из нуля при $\varepsilon>0$. Устойчивость будем понимать в метрике фазо-

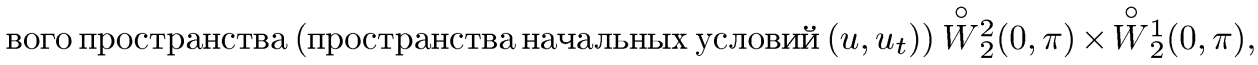
где $\stackrel{\circ}{W} \underset{2}{2}, \stackrel{\circ}{W} \underset{2}{1}$ - соболевские пространства функций, удовлетворяюших нулевым граничным условиям Дирихле. Использование понятия фазового пространства позволяет практически без изменений переносить на бесконечномерный случай основные положения теории нелинейных колебаний.

Предположим, что при условиях (4), (5) краевая задача (1) имеет некоторое нетривиальное квазипериодическое по $t$ решение

$$
u=u(\varphi, x, \varepsilon), \quad \varphi=\sigma(\varepsilon) t+c,
$$

где

$$
\sigma(\varepsilon)=\left(\left(1+\varepsilon \delta_{1}\right) \omega_{n_{1}},\left(1+\varepsilon \delta_{2}\right) \omega_{n_{2}}, \ldots,\left(1+\varepsilon \delta_{m}\right) \omega_{n_{m}}\right), \quad c=\left(c_{1}, \ldots, c_{m}\right) .
$$

Тогда, как нетрудно видеть, фигурирующая в (6) $2 \pi$-периодическая по векторному аргументу $\varphi$ функция $u(\varphi, x, \varepsilon)$ с необходимостью удовлетворяет краевой задаче

$$
\begin{aligned}
L^{2}(\varepsilon) u+\varepsilon L(\varepsilon) u+[1+\varepsilon f(\varphi)] u & =a^{2} u_{x x}-u^{2} L(\varepsilon) u, \\
\left.u\right|_{x=0}=\left.u\right|_{x=\pi} & =0,
\end{aligned}
$$

где $L(\varepsilon)=\sum_{k=1}^{m}\left(1+\varepsilon \delta_{k}\right) \omega_{n_{k}} \partial / \partial \varphi_{k}$. Таким образом, интересующая нас проблема сводится, в первую очередь, к нахождению нетривиальных $2 \pi$-периодических по $\varphi$ решений задачи (7).

Асимптотику возможных решений краевой задачи (7) будем строить в виде рядов по нечетным степеням $\sqrt{\varepsilon}$ :

$$
u=\sqrt{\varepsilon} u_{0}(\varphi, x)+\varepsilon^{3 / 2} u_{1}(\varphi, x)+\cdots,
$$

где $u_{0}(\varphi, x)$ - решение линейной краевой задачи $\left(L_{0}=L(0)\right)$

$$
L_{0}^{2} u_{0}+u_{0}=a^{2} \partial^{2} u_{0} / \partial x^{2},\left.\quad u_{0}\right|_{x=0}=\left.u_{0}\right|_{x=\pi}=0 .
$$

Выбирая, далее, в качестве $u_{0}(\varphi, x)$ различные $2 \pi$-периодические по $\varphi$ решения задачи (9), в принщипе, можно найти все интересующие нас решения задачи (7). Однако ниже ограничимся анализом так называемых одномодовых периодических решений (8), поскольку, как будет показано в дальнейшем, только они могут порождать устойчивые квазипериодические решения (6) исходной краевой задачи (1).

Для построения асимптотики одномодовых квазипериодических решений (6) фиксируем произвольное натуральное $k_{0}, k_{0} \leqslant m$, и в качестве решения задачи (9) рассмотрим функцию

$$
u_{0}(\varphi, x)=\left[\xi_{k_{0}} \exp \left(i \varphi_{k_{0}}\right)+\bar{\xi}_{k_{0}} \exp \left(-i \varphi_{k_{0}}\right)\right] \sin n_{k_{0}} x
$$


где $\xi_{k_{0}}$ - некоторая неизвестная (подлежащая определению) комплексная постоянная. Подставляя, далее, равенства (8), (10) в (7) и приравнивая коэффициенты при $\varepsilon^{3 / 2}$, для нахождения $u_{1}(\varphi, x)$ получаем линейную неоднородную краевую задачу

$$
L_{0}^{2} u_{1}+u_{1}=a^{2} \partial^{2} u_{1} / \partial x^{2}+g(\varphi, x),\left.\quad u_{1}\right|_{x=0}=\left.u_{1}\right|_{x=\pi}=0
$$

где

$$
g(\varphi, x)=2 \delta_{k_{0}} \omega_{n_{k_{0}}}^{2} u_{0}-f(\varphi) u_{0}-\omega_{n_{k_{0}}} \frac{\partial}{\partial \varphi_{k_{0}}}\left(u_{0}+\frac{u_{0}^{3}}{3}\right) .
$$

Анализ краевой задачи (11) сушественно опирается на тот факт, что в силу соотношений (10), (12) фигурируюшая в ней неоднородность $g$ представляет собой линейную комбинацию гармоник

$$
\begin{gathered}
\exp \left( \pm i p \varphi_{k_{0}}\right) \sin n_{k_{0}} x, \quad \exp \left( \pm i p \varphi_{k_{0}}\right) \sin 3 n_{k_{0}} x, \quad p=1,3 \\
\exp \left[ \pm i\left(2 \varphi_{s}+\varphi_{k_{0}}\right)\right] \sin n_{k_{0}} x, \quad \exp \left[ \pm i\left(2 \varphi_{s}-\varphi_{k_{0}}\right)\right] \sin n_{k_{0}} x \\
s=1, \ldots, m, \quad s \neq k_{0} .
\end{gathered}
$$

Поэтому для определения решения $u_{1}$ в классе тригонометрических многочленов аналогичной структуры необходимо и достаточно отсутствие в неоднородности (12) гармоник

$$
\exp \left( \pm i \varphi_{k_{0}}\right) \sin n_{k_{0}} x
$$

Добиться этого можно выбором имеющейся в запасе комплексной амплитуды $\xi_{k_{0}}$.

Действительно, приравнивая в (12) к нулю коэффициенты при гармониках (14), для определения $\xi_{k_{0}}$ приходим к уравнению

$$
-2 i \delta_{k_{0}} \omega_{n_{k_{0}}} \xi_{k_{0}}+\frac{i \alpha_{k_{0}}}{2 \omega_{n_{k_{0}}}} \bar{\xi}_{k_{0}}-\xi_{k_{0}}-\frac{3}{4}\left|\xi_{k_{0}}\right|^{2} \xi_{k_{0}}=0
$$

Полагая в $(15) \xi_{k_{0}}=\rho_{k_{0}} \exp \left(i \psi_{k_{0}}\right), \rho_{k_{0}}>0$, для нахождения $\rho_{k_{0}}, \psi_{k_{0}}$ получаем систему

$$
\frac{\alpha_{k_{0}}}{2 \omega_{n_{k_{0}}}} \cos 2 \psi_{k_{0}}=2 \delta_{k_{0}} \omega_{n_{k_{0}}}, \quad \frac{\alpha_{k_{0}}}{2 \omega_{n_{k_{0}}}} \sin 2 \psi_{k_{0}}=1+\frac{3}{4} \rho_{k_{0}}^{2} .
$$

Дальнейшая последовательность действий такова. Возведем левые и правые части уравнений этой системы в квадрат и сложим получившиеся равенства. В итоге убеждаемся, что

$$
\rho_{k_{0}}=\sqrt{\eta_{k_{0}}}, \quad \eta_{k_{0}}=\frac{4}{3}\left(\sqrt{\left.\frac{\alpha_{k_{0}}^{2}}{4 \omega_{n_{k_{0}}}^{2}}-4 \delta_{k_{0}}^{2} \omega_{n_{k_{0}}}^{2}-1\right)}\right.
$$

(положительность $\eta_{k_{0}}$ вытекает из условия $(5)$ при $k=k_{0}$ ). В качестве $\psi_{k_{0}}$, $\left|\psi_{k_{0}}\right|<\pi / 2$, выберем тот из корней первого уравнения (16), для которого

$$
\alpha_{k_{0}} \sin 2 \psi_{k_{0}}>0
$$

(см. второе уравнение (16)). 
После определения $\xi_{k_{0}}$ решение $u_{1}$ задачи (11) находится уже однозначно в виде линейной комбинации оставшихся гармоник (13) и, следовательно, приближенное одномодовое квазипериодическое решение

$$
u=\sqrt{\varepsilon} u_{0}(\varphi, x)+\varepsilon^{3 / 2} u_{1}(\varphi, x), \quad \varphi=\sigma(\varepsilon) t+c,
$$

краевой задачи (1) удается построить с точностью до $\varepsilon^{5 / 2}$ по невязке. Как будет показано ниже, в принципе, этого достаточно для доказательства существования соответствуюшего ему точного решения.

Перед формулировкой основного результата введем в рассмотрение числа $\eta_{k}$, $k=1, \ldots, m$, определяющиеся с помощью второго равенства из (17) (при замене в ней $k_{0}$ на $k$ ) и положительные в силу условий $(5)$.

ТЕОРЕМА. Пусть в дополнение к условиям (4), (5) выполняется неравенство

$$
\eta_{k_{0}}>(3 / 4) \max _{1 \leqslant s \leqslant m} \eta_{s}
$$

Тогда при всех достаточно малых $\varepsilon>0$ краевая задача (1) имеет әкспоненииально устойчивые одномодовые квазипериодические решения

$$
u= \pm u_{k_{0}}(\varphi, x, \varepsilon), \quad \varphi=\sigma(\varepsilon) t+c .
$$

Здесь $2 \pi$-периодическая по ч функиия $u_{k_{0}}$ такова, что

$$
u_{k_{0}}=\sqrt{\varepsilon}\left[\xi_{k_{0}} \exp \left(i \varphi_{k_{0}}\right)+\bar{\xi}_{k_{0}} \exp \left(-i \varphi_{k_{0}}\right)\right] \sin n_{k_{0}} x+\varepsilon^{3 / 2} \Delta_{k_{0}}(\varphi, x, \varepsilon),
$$

а для остатка $\Delta_{k_{0}}(\varphi, x, \varepsilon)$ справедлива оченка

$$
\max _{\varphi}\left\|\Delta_{k_{0}}\right\|_{W_{2}^{2}}^{\stackrel{\circ}{ }}+\max _{\varphi}\left\|L(\varepsilon) \Delta_{k_{0}}\right\|_{W_{2}^{1}} \leqslant N
$$

с не зависящей от в постоянной $N>0$.

Обращаем внимание на то, что, как следует из приводимого ниже доказательства теоремы, ее утверждение, касающееся существования квазипериодических решений (20) со свойствами (21), (22), сохраняется и при более слабых ограничениях. Достаточно вместо условия (19) потребовать отличие от нуля всех чисел

$$
R_{k_{0}, s}=\eta_{k_{0}}-(3 / 4) \eta_{s}, \quad s=1, \ldots, m
$$

Однако в этом случае рассматриваемые решения будут, вообше говоря, дихотомичными (с размерностью неустойчивых многообразий $s_{0}$, где $s_{0}$ - количество отрицательных среди чисел, описанных соотношением (23)). Отметим еще, что неравенство (19) заведомо выполняется, если номер $k_{0}$ удовлетворяет условию $\eta_{k_{0}}=\max _{1 \leqslant s \leqslant m} \eta_{s}$. Иными словами, пара одномодовых квазипериодических решений (20) с максимальной амплитудой $\rho_{k_{0}}$ всегда устойчива. 
2. Доказательство теоремы. Линеаризуем краевую задачу (1) на построенном вьше приближенном квазипериодическом решении (18). В результате получим линейную краевую задачу

$$
\begin{gathered}
h_{t t}+\varepsilon h_{t}+[1+\varepsilon f(\varphi)] h=a^{2} h_{x x}-\varepsilon a_{1}(\varphi, x, \varepsilon) h-\varepsilon a_{2}(\varphi, x, \varepsilon) h_{t}, \\
\left.h\right|_{x=0}=\left.h\right|_{x=\pi}=0
\end{gathered}
$$

с квазипериодическими коэффициентами, в которой

$$
a_{1}(\varphi, x, 0)=2 u_{0}(\varphi, x) L_{0}\left(u_{0}(\varphi, x)\right), \quad a_{2}(\varphi, x, 0)=u_{0}^{2}(\varphi, x) .
$$

Как известно (см., например, [6]), основной этап доказательства существования у краевой задачи (1) точного квазипериодического решения с асимптотикой (18) состоит в исследовании свойств устойчивости решений линейной краевой задачи (24) и в обратимости соответствуюшего ей дифференциального оператора (в подходящем пространстве $2 \pi$-периодических по $\varphi$ функций). Обе эти проблемы решаются с помошью алгоритма исследования устойчивости из [7], адаптированного в [8] для телеграфных уравнений. Описание указанного алгоритма приводится ниже.

Начнем с расчета характеристических показателей задачи (24), отвечающих модам $\sin n_{k} x, k=1, \ldots, m$. В соответствии с развитой в [8] методикой подставим в (24)

$$
\begin{gathered}
h=\left[V_{0, k}(\varphi, x)+\varepsilon V_{1, k}(\varphi, x)\right] \exp \left(\varepsilon D_{k} t\right), \\
V_{j, k}=\left[w_{j, k}, \bar{w}_{j, k}\right], \quad j=0,1, \quad w_{0, k}=\exp \left(i \varphi_{k}\right) \sin n_{k} x,
\end{gathered}
$$

где функщия $w_{1, k}$, являюшаяся тригонометрическим полиномом переменной $\varphi$, и элементы постоянной матрицы

$$
D_{k}=\left(\begin{array}{cc}
d_{1, k} & d_{2, k} \\
\bar{d}_{2, k} & \bar{d}_{1, k}
\end{array}\right)
$$

подлежат определению. Приравнивая коэффициенты при $\varepsilon$, для нахождения $w_{1, k}$ получим аналогичную (11) краевую задачу, в которой неоднородность $g$ следует заменить на

$$
\begin{aligned}
g_{k}= & -a_{1}(\varphi, x, 0) w_{0, k}-a_{2}(\varphi, x, 0) L_{0}\left(w_{0, k}\right)-L_{0}\left(w_{0, k}\right)-f(\varphi) w_{0, k} \\
& +2 \delta_{k} \omega_{n_{k}}^{2} w_{0, k}-2 i \omega_{n_{k}}\left(w_{0, k} d_{1, k}-\bar{w}_{0, k} \bar{d}_{2, k}\right) .
\end{aligned}
$$

Дальнейший анализ стандартен: сначала, приравнивая в (28) к нулю коэффициенты при гармониках $\exp \left( \pm i \varphi_{k}\right) \sin n_{k} x$, находим неизвестные постоянные $d_{1, k}$, $d_{2, k}$, для которых с учетом формул (25) выводим равенства

$$
\begin{aligned}
2 d_{1, k} & =-1-\eta_{k_{0}}-2 i \delta_{k} \omega_{n_{k}}, & 2 d_{2, k} & =i \alpha_{k} /\left(2 \omega_{n_{k}}\right), \quad k \neq k_{0}, \\
2 d_{1, k_{0}} & =-1-(3 / 2) \eta_{k_{0}}-2 i \delta_{k_{0}} \omega_{n_{k_{0}}}, & 2 d_{2, k_{0}} & =i \alpha_{k_{0}} /\left(2 \omega_{n_{k_{0}}}\right)-(3 / 4) \eta_{k_{0}}
\end{aligned}
$$


после этого решение $w_{1, k}$ получившейся краевой задачи однозначно определяем в виде тригонометрического многочлена аналогичной $u_{1}$ структуры при $k=k_{0}$ или линейной комбинации гармоник

$$
\begin{array}{ll}
\exp \left[i\left(\varphi_{k} \pm 2 \varphi_{k_{0}}\right)\right] \sin n_{k} x, & \exp \left[i\left(\varphi_{k} \pm 2 \varphi_{k_{0}}\right)\right] \sin \left(n_{k}+2 n_{k_{0}}\right) x, \\
\exp \left[i\left(\varphi_{k} \pm 2 \varphi_{k_{0}}\right)\right] \sin \left(n_{k}-2 n_{k_{0}}\right) x, & \exp \left(i \varphi_{k}\right) \sin \left(n_{k} \pm 2 n_{k_{0}}\right) x, \\
\exp \left[i\left(\varphi_{k} \pm 2 \varphi_{r}\right)\right] \sin n_{k} x, & r=1, \ldots, m, \quad r \neq k, k_{0},
\end{array}
$$

при $k \neq k_{0}$. Подчеркнем, что в последнем случае возможность нахождения $w_{1, k}$ в требуемом виде обеспечивают условия нерезонансности

$$
\begin{array}{rr}
\omega_{p}^{2}-\omega_{p \pm 2 n}^{2} \neq 0, \quad\left(\omega_{p}+2 \omega_{n}\right)^{2}-\omega_{p \pm 2 n}^{2} \neq 0, \\
\left(\omega_{p}-2 \omega_{n}\right)^{2}-\omega_{p \pm 2 n}^{2} \neq 0, \quad\left(\omega_{p} \pm 2 \omega_{n}\right)^{2}-\omega_{p}^{2} \neq 0,
\end{array}
$$

справедливые при всех натуральных $n, p, n \neq p$ (их обоснование содержится, например, в [9]).

При $p=1,2, \ldots, p \neq n_{k}, k=1, \ldots, m$, подставим в краевую задачу $(24)$ представление

$$
h=\exp \left[\left(\varepsilon \mu_{p}+i \omega_{p}\right) t\right]\left(\sin p x+\varepsilon h_{p}(\varphi, x)\right),
$$

где $h_{p}$ - тригонометрический многочлен переменной $\varphi$, a $\mu_{p}$ - подлежащая определению комплексная постоянная. В результате, приравнивая коэффициенты при $\varepsilon$, для $h_{p}$ получаем краевую задачу

$$
\begin{gathered}
\left(L_{0}^{2}+2 i \omega_{p} L_{0}-\omega_{p}^{2}-a^{2} \frac{\partial^{2}}{\partial x^{2}}\right) h_{p}+h_{p}=G_{p}(\varphi, x) \\
\left.h_{p}\right|_{x=0}=\left.h_{p}\right|_{x=\pi}=0
\end{gathered}
$$

с правой частью

$$
G_{p}(\varphi, x)=-\left[a_{1}(\varphi, x, 0)+i \omega_{p} a_{2}(\varphi, x, 0)+i \omega_{p}+f(\varphi)+2 i \omega_{p} \mu_{p}\right] \sin p x
$$

Далее, имеющуюся в запасе постоянную $\mu_{p}$ выберем из условия равенства нулю коэффициента при $\sin p x$ в неоднородности (34), что приводит к формуле

$$
2 \mu_{p}=-1-\eta_{k_{0}}
$$

После определения $\mu_{p}$ функция (34) приобретает вид

$$
\begin{aligned}
G_{p}= & \exp \left(2 i \varphi_{k_{0}}\right)\left[v_{1, p}^{+} \sin p x+v_{2, p}^{+} \sin \left(p+2 n_{k_{0}}\right) x+v_{3, p}^{+} \sin \left(p-2 n_{k_{0}}\right) x\right] \\
& +\exp \left(-2 i \varphi_{k_{0}}\right)\left[v_{1, p}^{-} \sin p x+v_{2, p}^{-} \sin \left(p+2 n_{k_{0}}\right) x+v_{3, p}^{-} \sin \left(p-2 n_{k_{0}}\right) x\right] \\
& +v_{4, p}^{+} \sin \left(p+2 n_{k_{0}}\right) x+v_{4, p}^{-} \sin \left(p-2 n_{k_{0}}\right) x \\
& +\sum_{s=1}^{m}\left[v_{4+s, p}^{+} \exp \left(2 i \varphi_{s}\right)+v_{4+s, p}^{-} \exp \left(-2 i \varphi_{s}\right)\right] \sin p x .
\end{aligned}
$$


Поэтому для решения $h_{p}$ задачи (33) получается аналогичная формула, в которой коэффициенты $v_{j, p}^{ \pm}, j=1, \ldots, m+4$, следует заменить на $\tilde{v}_{j, p}^{ \pm}$, где

$$
\begin{array}{ll}
\tilde{v}_{1, p}^{ \pm}=v_{1, p}^{ \pm} /\left[\omega_{p}^{2}-\left(\omega_{p} \pm 2 \omega_{n_{k_{0}}}\right)^{2}\right], & \tilde{v}_{2, p}^{ \pm}=v_{2, p}^{ \pm} /\left[\omega_{p+2 n_{k_{0}}}^{2}-\left(\omega_{p} \pm 2 \omega_{n_{k_{0}}}\right)^{2}\right] \\
\tilde{v}_{3, p}^{ \pm}=v_{3, p}^{ \pm} /\left[\omega_{p-2 n_{k_{0}}}^{2}-\left(\omega_{p} \pm 2 \omega_{n_{k_{0}}}\right)^{2}\right], & \tilde{v}_{4, p}^{ \pm}=v_{4, p}^{ \pm} /\left[\omega_{p \pm 2 n_{k_{0}}}^{2}-\omega_{p}^{2}\right] \\
\tilde{v}_{4+s, p}^{ \pm}=v_{4+s, p}^{ \pm} /\left[\omega_{p}^{2}-\left(\omega_{p} \pm 2 \omega_{n_{s}}\right)^{2}\right], & s=1, \ldots, m .
\end{array}
$$

Отметим, что, как и в предыдущем случае, отличие от нуля соответствующих знаменателей обеспечивают неравенства (31). Более того, поскольку после деления на $\omega_{p}$ левые части этих неравенств стремятся при $p \rightarrow \infty$ к ненулевым конечным пределам, то справедливы оценки

$$
\left|\tilde{v}_{j, p}^{ \pm}\right| \leqslant N, \quad j=1, \ldots, m+4
$$

здесь и ниже через $N, N_{1}$ и т.д. обозначаем различные универсальные (не зависяшие от $p, \varepsilon$ ) положительные постоянные, точные значения которых несущественны.

На следуюшем этапе доказательства обоснуем описанный выше алгоритм асимптотического вычисления характеристических показателей краевой задачи (24). Введем новые переменные $h_{1}=h_{t}, h_{2}=B h$, где $B$ - арифметический квадратный корень из оператора $-a^{2} d^{2} / d x^{2}+I$ с граничными условиями Дирихле, и перейдем обычным образом от уравнения (24) к системе первого порядка в $\stackrel{\circ}{W} \underset{2}{1} \times \stackrel{\circ}{W} \underset{2}{1}$. Выполним затем в получившейся системе замену, исходя из формулы

$$
h=\sum_{k=1}^{m}\left(V_{0, k}+\varepsilon V_{1, k}\right) z_{k}+\sum_{\substack{p \geqslant 1 \\ p \neq n_{k}, k=1, \ldots, m}} \omega_{p}^{-2}\left\{\left[\sin p x+\varepsilon h_{p}\right] y_{p}+\left[\sin p x+\varepsilon \bar{h}_{p}\right] \bar{y}_{p}\right\},
$$

где

$$
z_{k}=\operatorname{colon}\left(z_{k}^{0}, \bar{z}_{k}^{0}\right), \quad y=\operatorname{colon}\left(z_{1}^{0}, \bar{z}_{1}^{0}, \ldots, z_{m}^{0}, \bar{z}_{m}^{0}, y_{1}, \bar{y}_{1}, \ldots, y_{p}, \bar{y}_{p}, \ldots\right) \in l_{2} .
$$

Последнее означает, что при дифференцировании переменных $z_{k}=z_{k}(t), y_{p}=$ $y_{p}(t)$ по $t$ следует считать

$$
\dot{z}_{k}=\varepsilon D_{k} z_{k}, \quad k=1, \ldots, m, \quad \dot{y}_{p}=\left(i \omega_{p}+\varepsilon \mu_{p}\right) y_{p}
$$

где $D_{k}$ - матрицы (27), а $\mu_{p}$ - числа (35). Заметим, далее, что из способа построения и свойств входящих в (37) функций (см. равенства (26), (32) и оценки (36)) вытекают следующие факты. Во-первых, формула (37) индуцирует ограниченный равномерно по $\varphi, \varepsilon$ (вместе со своим обратным) оператор, действующий из $l_{2}$ в $\stackrel{\circ}{W} \underset{2}{1} \times \stackrel{\circ}{W} \underset{2}{1}$. Во-вторых, результатом проведения указанной замены в (24) служит система в $l_{2}$ :

$$
\dot{y}=\Lambda_{0}(\varepsilon) y+\varepsilon^{2} \Lambda_{1}(\varphi, \varepsilon) y .
$$


Здесь

$$
\Lambda_{0}=\operatorname{diag}\left\{\varepsilon D_{1}, \ldots, \varepsilon D_{m}, \ldots, i \omega_{p}+\varepsilon \mu_{p},-i \omega_{p}+\varepsilon \mu_{p}, \ldots\right\},
$$

а линейный $2 \pi$-периодический по $\varphi$ оператор $\Lambda_{1}$ ограничен равномерно по $\varphi, \varepsilon$.

Из структуры системы (38) следует, что свойства ее устойчивости определяются по расположению спектра оператора (39), а значит, в конечном итоге - по расположению собственных значений матрищ (27). Из (29), (30) следует, что матрица $D_{k_{0}}$ является гурвищевой, а матрищы $D_{k}$ при $k \neq k_{0}$ будут таковыми при

$$
\eta_{k_{0}}>(3 / 4) \eta_{k}, \quad k=1, \ldots, m, \quad k \neq k_{0} .
$$

Остается добавить, что справедливость всех неравенств (40) обеспечивает предполагаемое выше условие (19).

Завершаюший этап обоснования теоремы состоит в доказательстве существования у краевой задачи (7) $2 \pi$-периодического по $\varphi$ решения с построенной вьше асимптотикой и проводится по стандартной схеме. Положим в (7)

$$
u=\sqrt{\varepsilon} u_{0}(\varphi, x)+\varepsilon^{3 / 2} u_{1}(\varphi, x)+\varepsilon^{3 / 2} h,
$$

где функции $u_{0}, u_{1}$ взяты из (18). В результате приходим к уравнению

$$
\Pi(\varepsilon) h=\varepsilon F(\varphi, x, \varepsilon, h, L(\varepsilon) h),
$$

где

$\Pi(\varepsilon) h=L^{2}(\varepsilon) h+\varepsilon L(\varepsilon) h+[1+\varepsilon f(\varphi)] h-a^{2} h_{x x}+\varepsilon a_{1}(\varphi, x, \varepsilon) h+\varepsilon a_{2}(\varphi, x, \varepsilon) L(\varepsilon) h$,

а гладкая по совокупности переменных $2 \pi$-периодическая по $\varphi$ функция $F(\varphi, x, \varepsilon$, $u, v)$ такова, что

$$
\begin{gathered}
\max _{\varphi}\|F(\varphi, x, \varepsilon, 0,0)\|_{W_{W_{2}^{1}}^{\circ}} \leqslant N_{1}, \\
\max _{\varphi}\left\|F\left(\varphi, x, \varepsilon, u_{1}, v_{1}\right)-F\left(\varphi, x, \varepsilon, u_{2}, v_{2}\right)\right\|_{W_{2}^{1}}^{\circ} \leqslant N_{2} \varepsilon\left(\left|u_{1}-u_{2}\right|+\left|v_{1}-v_{2}\right|\right),
\end{gathered}
$$

где $N_{2}=N_{2}(R), R=\max \left\{\left|u_{j}\right|,\left|v_{j}\right|, j=1,2\right\}$.

Введем в рассмотрение банаховы пространства $E_{j}, j=1,2$, состоящие из $2 \pi$-периодических и непрерьвных по $\varphi$ в метрике $\stackrel{\circ}{W}_{2}^{j}(0, \pi), j=1,2$, функций $u(\varphi, x)$. Нормы в этих пространствах определим формулами

$$
\|u\|_{E_{j}}=\max _{\varphi}\|u(\varphi, x)\|_{W_{2}^{j}}, \quad j=1,2 .
$$

Рассмотрим, далее, уравнение

$$
\Pi(\varepsilon) h=g(\varphi, x)
$$

с произвольной неоднородностью $g \in E_{1}$. Справедливо следующее утверждение. 
ЛЕмма. Уравнение (46) имеет при любой правой части $g \in E_{1}$ единственное решение $u=u_{g}(\varphi, x, \varepsilon) \in E_{2}$, удовлетворяющее неравенству

$$
\left\|u_{g}\right\|_{E_{2}}+\left\|L(\varepsilon) u_{g}\right\|_{E_{1}} \leqslant \frac{N_{3}}{\varepsilon}\|g\|_{E_{1}} .
$$

ДоКАЗАТЕЛЬСТВо. Как и при обосновании алгоритма исследования устойчивости, введем новые переменные $h_{1}=L(\varepsilon) h, h_{2}=B h$ и перейдем от уравнения (46) к системе в $E_{1} \times E_{1}$. Вьполним, далее, в этой системе замену, исходя из формулы $(37)$ и равенств

$$
L(\varepsilon) z_{k}=\varepsilon D_{k} z_{k}, \quad k=1, \ldots, m, \quad L(\varepsilon) y_{p}=\left(i \omega_{p}+\varepsilon \mu_{p}\right) y_{p} .
$$

В итоге приходим к уравнению для $y \in l_{2}$, имеюшему вид

$$
L(\varepsilon) y=\Lambda_{0}(\varepsilon) y+\varepsilon^{2} \Lambda_{1}(\varphi, \varepsilon) y+\tilde{g}(\varphi, \varepsilon) .
$$

Здесь, как и раньше, $L(\varepsilon) y=\sum_{k=1}^{m}\left(1+\varepsilon \delta_{k}\right) \omega_{n_{k}} \frac{\partial y}{\partial \varphi_{k}}, \Lambda_{0}, \Lambda_{1}$ - операторы из (38), $\tilde{g}(\varphi, \varepsilon)$ - неоднородность из класса $C\left(l_{2}\right)$, где $C\left(l_{2}\right)$ - банахово пространство непрерывных в метрике $l_{2}$ и $2 \pi$-периодических по $\varphi$ функций $y(\varphi)$ с нормой

$$
\|y\|_{C\left(l_{2}\right)}=\max _{\varphi}\|y(\varphi)\|_{l_{2}} \text {. }
$$

Итак, проблема обоснования леммы свелась к нахождению решения $y(\varphi, \varepsilon)$ уравнения (48), принадлежащего классу $C\left(l_{2}\right)$. Анализ этого уравнения разбивается, в свою очередь, на два этапа.

На первом этапе рассмотрим вспомогательное уравнение

$$
L(\varepsilon) y=\Lambda_{0}(\varepsilon) y+\tilde{g}(\varphi, \varepsilon)
$$

и заметим, что, как следует из явного вида оператора $\Lambda_{0}(\varepsilon)$, оно имеет единственное решение $y=y(\varphi, \varepsilon) \in C\left(l_{2}\right)$, задаюшееся равенством

$$
y(\varphi, \varepsilon)=\int_{-\infty}^{0} \exp \left[-\Lambda_{0}(\varepsilon) s\right] \tilde{g}(\sigma(\varepsilon) s+\varphi, \varepsilon) d s .
$$

Более того, из формулы (49) очевидным образом вытекает оценка

$$
\|y\|_{C\left(l_{2}\right)} \leqslant \frac{N_{4}}{\varepsilon}\|\tilde{g}\|_{C\left(l_{2}\right)} .
$$

На втором этапе, рассматривая второе и третье слагаемые правой части уравнения (48) как неоднородность и применяя формулу (49), приходим к линейному неоднородному интегральному уравнению в $C\left(l_{2}\right)$. Обозначим, далее, через $\widetilde{\Pi}: C\left(l_{2}\right) \rightarrow C\left(l_{2}\right)$ оператор, порожденньй правой частью получившегося уравнения. Из оценки $(50)$ и свойств $\Lambda_{1}(\varphi, \varepsilon)$ следует, что оператор Пٓ является сжимаюшим (с константой сжатия порядка $\varepsilon$ ). Тем самым, уравнение (48) имеет единственное решение $y(\varphi, \varepsilon) \in C\left(l_{2}\right)$, для которого сохраняется аналогичная (50) оценка. 
И наконец, возврашаясь к исходному уравнению (46), т.е. обрашая сделанную ранее замену, убеждаемся в существовании и единственности его решения $u_{g} \in E_{2} \mathrm{c}$ требуемыми свойствами. Лемма доказана.

Для завершения доказательства теоремы перейдем от (42) к интегральному уравнению в $E_{2}$, обрашая оператор (43). Неравенства (44), (45), (47) позволяют применить к нему принцип сжимаюших отображений и определить функцию $h(\varphi, x, \varepsilon) \in E_{2}$, удовлетворяющую оценке

$$
\|h\|_{E_{2}}+\|L(\varepsilon) h\|_{E_{1}} \leqslant N_{5} .
$$

Подставляя, далее, эту функцию в (41), получаем решение (21) краевой задачи (7) со свойствами (22). Остается добавить, что поскольку исходная краевая задача (1) инвариантна относительно замены $u \rightarrow-u$, то одному и тому же решению (21) соответствует пара квазипериодических решений (20). Устойчивость же этих решений вытекает из установленных выше свойств системы (38). Теорема доказана.

3. Об устойчивости многомодовых решений. Для получения более полного представления о динамике краевой задачи (1) воспользуемся методом квазинормальньх форм [10]. С этой целью положим в ней

$$
u=\sqrt{\varepsilon} u_{0}(t, \varphi, \tau, x)+\varepsilon^{3 / 2} u_{1}(t, \varphi, \tau, x)+\cdots, \quad \tau=\varepsilon t,
$$

где

$$
\begin{gathered}
u_{0}=u_{0,0}(\tau, \varphi, x)+u_{0,1}(t, \tau, x), \\
u_{0,0}(\tau, \varphi, x)=\sum_{k=1}^{m}\left[\xi_{n_{k}}(\tau) \exp \left(i \varphi_{k}\right)+\bar{\xi}_{n_{k}}(\tau) \exp \left(-i \varphi_{k}\right)\right] \sin n_{k} x, \\
u_{0,1}(t, \tau, x)=\sum_{\substack{p=1 \\
p \neq n_{k}, k=1, \ldots, m}}^{\infty}\left[\xi_{p}(\tau) \exp \left(i \omega_{p} t\right)+\bar{\xi}_{p}(\tau) \exp \left(-i \omega_{p} t\right)\right] \sin p x,
\end{gathered}
$$

а комплексные амплитуды $\xi_{s}, s \geqslant 1$, таковы, что сходится ряд с обшим членом $\omega_{s}^{4}\left|\xi_{s}\right|^{2}$ (в этом случае $u_{1} \in \stackrel{\stackrel{2}{W}}{2}$ по переменной $x$ ). Приравнивая затем коэффициенты при $\varepsilon^{3 / 2}$, для определения $u_{2}$ получаем краевую задачу

$$
\left(\frac{\partial}{\partial t}+L_{0}\right)^{2} u_{2}+u_{2}-a^{2} \frac{\partial^{2} u_{2}}{\partial x^{2}}=G,\left.\quad u_{2}\right|_{x=0}=\left.u_{2}\right|_{x=\pi}=0
$$

в которой

$$
\begin{aligned}
G= & 2 \sum_{k=1}^{m} \delta_{k} \omega_{n_{k}}^{2}\left[\xi_{n_{k}}(\tau) \exp \left(i \varphi_{k}\right)+\bar{\xi}_{n_{k}}(\tau) \exp \left(-i \varphi_{k}\right)\right] \sin n_{k} x \\
& -f(\varphi) u_{0}-\left(\frac{\partial}{\partial t}+L_{0}\right)\left(2 \frac{\partial u_{0}}{\partial \tau}+u_{0}+\frac{u_{0}^{3}}{3}\right)
\end{aligned}
$$


Обратим внимание на то, что необходимым условием разрешимости краевой задачи (51) в классе формальных тригонометрических рядов переменных $t, \varphi$ является отсутствие в ее правой части (52) слагаемых, пропорциональных

$$
\begin{gathered}
\exp \left( \pm i \varphi_{k}\right) \sin n_{k} x, \quad k=1, \ldots, m, \quad \exp \left( \pm i \omega_{p} t\right) \sin p x \\
p \geqslant 1, \quad p \neq n_{k}, \quad k=1, \ldots, m
\end{gathered}
$$

Приравнивая упомянутые слагаемые к нулю, для нахождения неизвестных амплитуд $\xi_{s}, s \geqslant 1$, приходим к системе обыкновенных уравнений (дифференцирование проводим по $\tau$ )

$$
\begin{aligned}
2 \dot{\xi}_{n_{k}}= & -\left(2 \delta_{k} i \omega_{n_{k}}+1\right) \xi_{n_{k}}+\frac{i \alpha_{k}}{2 \omega_{n_{k}}} \bar{\xi}_{n_{k}} \\
& -\frac{3}{4}\left|\xi_{n_{k}}\right|^{2} \xi_{n_{k}}-\xi_{n_{k}} \sum_{\substack{s=1 \\
s \neq n_{k}}}^{\infty}\left|\xi_{s}\right|^{2}, \quad k=1, \ldots, m, \\
2 \dot{\xi}_{p}= & -\xi_{p}-\frac{3}{4}\left|\xi_{p}\right|^{2} \xi_{p}-\xi_{p} \sum_{\substack{s=1 \\
s \neq p}}^{\infty}\left|\xi_{s}\right|^{2}, \quad p \geqslant 1, \quad p \neq n_{k}, \quad k=1, \ldots, m,
\end{aligned}
$$

которая и является искомой квазинормальной формой задачи (1).

Из структуры уравнений (54) следует, что все амплитуды $\xi_{p}, p \neq n_{k}, k=$ $1, \ldots, m$, стремятся к нулю при $\tau \rightarrow \infty$. Поэтому уместно перейти от полной системы $(53),(54)$ к системе на устойчивом инвариантном многообразии $\left\{\xi_{p}=0\right.$, $\left.p \neq n_{k}, k=1, \ldots, m\right\}$, которая имеет вид

$$
\begin{gathered}
2 \dot{\xi}_{n_{k}}=-\left(2 \delta_{k} i \omega_{n_{k}}+1\right) \xi_{n_{k}}+\frac{i \alpha_{k}}{2 \omega_{n_{k}}} \bar{\xi}_{n_{k}}-\frac{3}{4}\left|\xi_{n_{k}}\right|^{2} \xi_{n_{k}}-\xi_{n_{k}} \sum_{\substack{s=1 \\
s \neq k}}^{m}\left|\xi_{n_{s}}\right|^{2} \\
k=1, \ldots, m .
\end{gathered}
$$

Фиксируем произвольно набор натуральных чисел

$$
1 \leqslant k_{1}<k_{2}<\cdots<k_{r} \leqslant m
$$

и предположим, что система (55) имеет состояние равновесия с компонентами

$$
\xi_{n_{k_{j}}} \neq 0, \quad j=1, \ldots, r, \quad \xi_{n_{k}}=0, \quad n_{k} \neq n_{k_{j}}
$$

экспоненциально устойчивое или дихотомичное. Тогда, используя развитую в п. 2 технику, можно показать, что при выполнении определенных условий нерезонансности между собственными частотами $\omega_{n}, n \geqslant 1$ (см. [8]), исходная краевая задача (1) имеет квазипериодическое решение (6) с теми же свойствами устойчивости. 
Асимптотика этого решения задается равенством (8), в котором в качестве $u_{0}(\varphi, x)$ следует взять функцию

$$
u_{0}(\varphi, x)=\sum_{j=1}^{r}\left[\xi_{n_{k_{j}}} \exp \left(i \varphi_{k_{j}}\right)+\bar{\xi}_{n_{k_{j}}} \exp \left(-i \varphi_{k_{j}}\right)\right] \sin n_{k_{j}} x .
$$

Такое решение естественно назвать $r$-модовым.

Поскольку вопрос о существовании и устойчивости одномодовых квазипериодических решений (6) уже рассмотрен, то будем считать, что в (56) $r \geqslant 2$. Далее, для нахождения интересуюших нас состояний равновесия (57) системы (55) положим в ней $\xi_{n_{k}}=0$ при $n_{k} \neq n_{k_{j}}, \xi_{n_{k_{j}}}=\rho_{k_{j}} \exp \left(i \psi_{k_{j}}\right), j=1, \ldots, r$. В результате для переменных $\eta_{j}=\rho_{k_{j}}^{2}, \theta_{j}=2 \psi_{k_{j}}$ получим систему

$$
\begin{aligned}
& \dot{\eta}_{j}=\frac{\alpha_{k_{j}}}{2 \omega_{n_{k_{j}}}} \eta_{j} \sin \theta_{j}-\eta_{j}\left[1+\frac{3}{4} \eta_{j}+\sum_{\substack{s=1 \\
s \neq j}}^{r} \eta_{s}\right], \\
& \dot{\theta}_{j}=-2 \delta_{k_{j}} \omega_{n_{k_{j}}}+\frac{\alpha_{k_{j}}}{2 \omega_{n_{k_{j}}}} \cos \theta_{j}, \quad j=1, \ldots, r .
\end{aligned}
$$

Анализ системы (58) проведем при дополнительных условиях

$$
\frac{4}{4 m-1} \sum_{s=1}^{m} \varkappa_{s}-\varkappa_{j}>0, \quad j=1, \ldots, r,
$$

где $\varkappa_{j}=\sqrt{\alpha_{k_{j}} /\left(4 \omega_{n_{k_{j}}}^{2}\right)-4 \delta_{k_{j}}^{2} \omega_{n_{k_{j}}}^{2}}-1, j=1, \ldots, r$. Неравенства (59), как нетрудно увидеть, обеспечивают существование у системы состояния равновесия $\eta_{j}=\eta_{j}^{0}, \theta_{j}=\theta_{j}^{0}, j=1, \ldots, r$, где

$$
\eta_{j}^{0}=4\left(\frac{4}{4 m-1} \sum_{s=1}^{m} \varkappa_{s}-\varkappa_{j}\right)>0, \quad j=1, \ldots, r
$$

а $\theta_{j}^{0}, j=1, \ldots, r,-$ корни уравнений

$$
\frac{\alpha_{k_{j}}}{2 \omega_{n_{k_{j}}}} \cos \theta_{j}=2 \delta_{k_{j}} \omega_{n_{k_{j}}}, \quad j=1, \ldots, r,
$$

для которых выполняются условия

$$
\alpha_{k_{j}} \sin \theta_{j}^{0}>0, \quad j=1, \ldots, r .
$$

Обозначим, далее, через $C$ матрицу линеаризации системы (58) на указанном состоянии равновесия. Из структуры уравнений для $\theta_{j}$ вытекает, что $r$ ее собственных значений совпадают с числами

$$
-\frac{\alpha_{k_{j}}}{2 \omega_{n_{k_{j}}}} \sin \theta_{j}^{0}, \quad j=1, \ldots, r
$$


а значит, являются отрицательными (см. (61)). Остальные же $r$ собственных значений матрищы $C$ определяются из уравнения

$$
\operatorname{det}\left(\lambda C_{1}+C_{2}\right)=0
$$

где

$$
C_{1}=\operatorname{diag}\left\{1 / \eta_{1}^{0}, 1 / \eta_{2}^{0}, \ldots, 1 / \eta_{r}^{0}\right\}, \quad C_{2}=\left(\begin{array}{ccccc}
3 / 4 & 1 & \ldots & \ldots & 1 \\
1 & 3 / 4 & 1 & \ldots & 1 \\
\ldots \ldots & \ldots & \ldots & \ldots & \ldots \\
1 & \ldots & \ldots & 1 & 3 / 4
\end{array}\right)
$$

Покажем, что уравнение (62) имеет хотя бы один положительный корень. Предположим сначала, что найдутся такие индексы $j_{1} \neq j_{2}$, для которых $\eta_{j_{1}}^{0}=\eta_{j_{2}}^{0}$. Тогда это уравнение имеет корень $\lambda=\eta_{j_{1}}^{0} / 4$, поскольку одинаковьми становятся строки матрицы $\left(\eta_{j_{1}}^{0} / 4\right) C_{1}+C_{2}$ с номерами $j_{1}$ и $j_{2}$. Поэтому предположим, что все числа (60) различны, а точнее говоря, без ограничения общности будем считать, что

$$
\eta_{1}^{0}<\eta_{2}^{0}<\cdots<\eta_{r}^{0}
$$

Обратимся к фигурирующей в уравнении (62) матрище $\lambda C_{1}+C_{2}$ и вычтем первую ее строку из всех остальных строк. После этого к первому столбцу получившейся матрицы прибавим все остальные столбцы, умноженные соответственно на $\left(\frac{\lambda}{\eta_{1}^{0}}-\frac{1}{4}\right) /\left(\frac{\lambda}{\eta_{j}^{0}}-\frac{1}{4}\right), j=2, \ldots, r$. В итоге уравнение $(62)$ преобразуется к виду

$$
P(\lambda) \equiv\left[1+\sum_{s=1}^{r} \frac{1}{\lambda / \eta_{s}^{0}-1 / 4}\right] \prod_{s=1}^{r}\left(\frac{\lambda}{\eta_{s}^{0}}-\frac{1}{4}\right)=0 .
$$

Заметим, далее, что из условий (63) вытекают равенства

$$
\operatorname{sign} P\left(\eta_{s} / 4\right)=(-1)^{r-s}, \quad s=1, \ldots, r .
$$

Это означает, что количество положительных корней уравнения (64) равно $r-1$.

Итак, установлено, что любое $r$-модовое квазипериодическое решение (6) краевой задачи (1) при $r \geqslant 2$ если и сушествует, то заведомо неустойчиво. Тем самым, ее аттракторы исчерпываются построенными в п. 2 одномодовыми квазипериодическими решениями.

4. Заключение. Интересно отметить, что все полученные для краевой задачи (1) факты сохраняются и в случае краевой задачи

$$
u_{t t}+\varepsilon u_{t}+[1+\varepsilon f(\varphi)] \sin u=a^{2} u_{x x},\left.\quad u\right|_{x=0}=\left.u\right|_{x=\pi}=0
$$

c “маятниковой” нелинейностью при выполнении прежних ограничений (4), (5). 
Действительно, динамику краевой задачи (65) описывает аналогичная (55) система

$$
\begin{gathered}
2 \dot{\xi}_{s}=-\left(1+2 \delta_{s} i \omega_{n_{s}}\right) \xi_{s}+\frac{i \alpha_{s}}{2 \omega_{n_{s}}} \bar{\xi}_{s}-\frac{i}{\omega_{n_{s}}}\left[\frac{3}{8}\left|\xi_{s}\right|^{2} \xi_{s}+\frac{1}{2} \xi_{s} \sum_{\substack{k=1 \\
k \neq s}}^{m}\left|\xi_{s}\right|^{2}\right], \\
s=1, \ldots, m .
\end{gathered}
$$

Фиксируем, далее, произвольное натуральное $k_{0}, k_{0} \leqslant m$, и заметим, что эта система имеет состояние равновесия

$$
\xi_{k_{0}}=\sqrt{\eta_{k_{0}}} \exp \left(i \psi_{k_{0}}\right), \quad \xi_{k}=0, \quad k \neq k_{0},
$$

где $\eta_{k_{0}}=\frac{8}{3}\left(\sqrt{\alpha_{k_{0}}^{2} / 4-\omega_{n_{k_{0}}}^{2}}-2 \delta_{k_{0}} \omega_{n_{k_{0}}}^{2}\right)>0$ (см. неравенство (5) при $\left.k=k_{0}\right)$, а $\psi_{k_{0}}-$ корень уравнения

$$
\frac{\alpha_{k_{0}}}{2} \cos 2 \psi_{k_{0}}=2 \delta_{k_{0}} \omega_{n_{k_{0}}}^{2}+\frac{3}{8} \eta_{k_{0}}
$$

удовлетворяющий условию $\alpha_{k_{0}} \sin 2 \psi_{k_{0}}>0$. Нетрудно также заметить, что условие устойчивости состояния равновесия (67) задается прежним неравенством (19). Добавим еще, что при некоторых дополнительных ограничениях система (66) может иметь и состояния равновесия с количеством ненулевых координат $r \geqslant 2$. Все они, однако, неустойчивы.

В заключение сопоставим полученные выше результаты с автоколебательным случаем. А именно, рассмотрим краевую задачу

$$
u_{t t}-\varepsilon u_{t}+u=a^{2} u_{x x}-u^{2} u_{t},\left.\quad u\right|_{x=0}=\left.u\right|_{x=\pi}=0,
$$

получающуюся из (1) при $f(\varphi)=0$ и замене слагаемого $\varepsilon u_{t}$ на $-\varepsilon u_{t}$. Как показано, например, в [9], в рамках задачи (68) реализуется так называемое явление буферности, состоящее в том, что при уменьшении $\varepsilon$ можно гарантировать существование для этой задачи любого наперед заданного конечного числа устойчивых циклов (периодических по $t$ решений). Точнее говоря, для каждого натурального $N$ найдется такое $\varepsilon_{N}>0$, что при всех $0<\varepsilon \leqslant \varepsilon_{N}$ краевая задача (68) имеет экспоненциально орбитально устойчивые циклы

$$
u=\sqrt{\varepsilon} u_{n}(\tau, x, \varepsilon), \quad d \tau / d t=\omega_{n}\left(1+\varepsilon \delta_{n}(\varepsilon)\right), \quad n=1,2, \ldots, N .
$$

Здесь достаточно гладкие по своим переменным функции $\delta_{n}(\varepsilon), u_{n}(\tau, x, \varepsilon), u_{n}(\tau+$ $2 \pi, x, \varepsilon) \equiv u_{n}(\tau, x, \varepsilon)$ таковы, что

$$
\delta_{n}(0)=0, \quad u_{n}(\tau, x, 0)=\frac{4}{\sqrt{3}} \cos \tau \sin n x .
$$

Недостатком краевой задачи (68) является то, что буферность в ней носит "дикий” характер: возбуждены все моды и бифурцируюших из нуля циклов излишне 
много (как установлено в [9], при $\varepsilon \rightarrow 0$ их количество имеет порядок $\varepsilon^{-1}$ ). С другой стороны, как показывает проделанный нами анализ, явлением буферности можно управлять. Рассмотрим вместо (68) недовозбужденную систему, т.е. краевую задачу

$$
u_{t t}+\varepsilon u_{t}+u=a^{2} u_{x x}-u^{2} u_{t},\left.\quad u\right|_{x=0}=\left.u\right|_{x=\pi}=0
$$

Если начать действовать на нее параметрической внешней силой (2), то можно добиться возбуждения только конечного числа мод с наперед заданными номерами $n_{1}<n_{2}<\cdots<n_{m}$. Более того, если положить, например, в (2), (4) $\alpha_{k}=\alpha \omega_{n_{k}}$, $\delta_{k}=\delta / \omega_{n_{k}}, k=1, \ldots, m$, где постоянные $\alpha, \delta$ связаны неравенством

$$
\alpha^{2} / 4-4 \delta^{2}-1>0
$$

то условия устойчивости (19) одномодовых квазипериодических решений (20) будут выполняться одновременно при всех $k_{0}=1, \ldots, m$. Иными словами, в рамках краевой задачи (1) наблюдается так называемое явление управляемой буфернос$m u$, т.е. за счет выбора параметров внешнего воздействия можно управлять в ней как количеством и номерами возбуждаемых мод, так и количеством возникающих при этом устойчивых квазипериодических режимов.

\section{Список литературы}

1. Боголюбов H. Н., Митропольский Ю. А. Асимптотические методы в теории нелинейных колебаний. М.: Наука, 1974.

2. Якубович B. А., Старжинский B. М. Линейные дифференциальные уравнения с периодическими коэффициентами и их приложения. М.: Наука, 1972.

3. Митропольский Ю. А., Мосеенков Б. И. Асимптотические решения уравнений в частных производных. Киев: Виша шк., 1976.

4. Фомин B. Н. Математическая теория параметрического резонанса в линейных распределенных системах. Л.: Изд-во ЛГУ, 1972.

5. Кубъикин E. П. Параметрический резонанс в системах с последействием при почти периодическом возмушении // Исследования по устойчивости и теории колебаний. Ярославль: Изд-во ЯГУ, 1978. С. 100-109.

6. Васильева А.Б., Кащенко С.А., Колесов Ю. С., Розов Н.Х. Бифуркация автоколебаний нелинейных параболических уравнений с малой диффузией // Матем. сб. 1986. T. 130. № 4. C. 488-499.

7. Колесов Ю. С., Майоров В. В. Новый метод исследования устойчивости решений линейных дифференциальных уравнений с близкими к постоянным почти периодическими коэффициентами // Дифференц. уравн. 1974. Т. 10. № 10. С. 1778-1788.

8. Колесов А. Ю. Устойчивость автоколебаний телеграфного уравнения, бифурцирующих из состояния равновесия // Матем. заметки. 1992. Т. 51. № 2. С. 59-65.

9. Колесов А.Ю., Мищенко Е.Ф., Розов Н.Х. Асимптотические методы исследования периодических решений нелинейных гиперболических уравнений // Тр. МИРАН. 1998. T. 222. C. $3-191$.

10. Колесов Ю. С. Асимптотика и устойчивость нелинейных параметрических колебаний сингулярно возмущенного телеграффного уравнения // Матем. сб. 1995. Т. 186. № 10. C. $57-72$. 